\title{
HISPANISMOS EN EL DICCIONARIO DE INGLÉS CRIOLLO DE COSTA RICA
}

\author{
Mario Portilla
}

\begin{abstract}
RESUMEN
El presente artículo presenta una discusión acerca de la posible inclusión de hispanismos en un diccionario dialectal plurilingüe de inglés criollo de Costa Rica -inglés estándar- español, el cual se encuentra en elaboración. Se consideran, como parte de la respuesta a este problema, los siguientes aspectos téorico-metodológicos: 1. La distinción clara entre las nociones de préstamo léxico, mezcla de códigos y cambio de códigos. 2. La pertinencia de la función estandarizadora que atañería al diccionario en cuestión, según la definición tipológico-lexicográfica que se le ha asignado. 3. La pertinencia funcional de los hispanismos para los destinatarios del diccionario.
\end{abstract}

\begin{abstract}
This article presents a discussion about the possible inclusion of words borrowed from Spanish in a dialectal Costa Rican Creole English -Standard English-Spanish dictionary. As a partial solution of this question, three theorethical and methodological aspects are considered: 1. The clear distinction between the concepts of lexical borrowing, code mixing, and code switching. 2 . The relevance of the standardization that concerns this dictionary, according to its lexicographical and tipological characterization. 3. The functional relevance of the Spanish loanwords to the dictionary-users.
\end{abstract}

\section{Introducción}

\subsection{El diccionario de inglés criollo de Costa Rica}

Actualmente se está desarrollando, en la Universidad de Costa Rica, un proyecto de investigación en lexicografía del inglés criollo de Costa Rica. Este proyecto incluye la elaboración de varios diccionarios, entre ellos un diccionario dialectal plurilingüe de inglés criollo -inglés estándar- español.

\subsection{Recolección de vocablos de origen español}

Hasta el momento, el trabajo con informantes ha dado como resultado la recolección de una considerable cantidad de términos de origen español. Estos vocablos han sido obtenidos de diversas formas durante las sesiones de trabajo: 
A. Estos han sido ofrecidos espontáneamente como respuesta lingüística a un determinado estímulo

A.a. o bien como el término de un determinado objeto o evento mostrado en una lámina:

kokíeta (coqueta), alfúombra (alfombra), masetíero (masetero), porsiána (persiana), kwáadro (cuadro), etc.;

A.b. o bien como equivalente de un vocablo dado en español, en respuesta a preguntas como la siguiente: ¿cómo diría usted en criollo cabanga ${ }^{1}$ ?:

kabáangga (cabanga), yúuka (yuca), piá piá (piapia ${ }^{2}$ ), chokáar (chocar), etc.

B. También, estos vocablos han surgido espontáneamente del informante durante las sesiones de trabajo, dentro de textos:

(1) Shi ful a práblm práblm. Mi a aid fram ar. Mi a eskondér fram ar.

ella estar-llena de problema problema. yo PRO esconder de ella. yo PRO esconder de ella

Ella es solo problemas. Me le estoy escondiendo. Me le estoy escondiendo.

(2) Di saluóng ful op a bénchi bénchi aal bout.

el salón estar-lleno INT de banco banco todo alrededor

El salón estaba lleno de un banquerío por todo lado.

C. Además, otros términos recogidos han sido sugeridos por el investigador (los cuales son avalados y reproducidos nuevamente por el informante)

C.a. o bien a partir de fuentes documentales de otras variedades de inglés criollo de la región, como, por ejemplo, del glosario de Holm 1978 del inglés criollo de Nicaragua (cf. bibliografía). Algunas preguntas que sirven de estímulo son las siguientes: ¿se usa en criollo la palabra "kalsetíng" (calcetín)? o ¿significa algo la palabra "báagos" (vagabundo)?;

C.b. o bien simplemente mencionando términos que se ofrecen como una posibilidad, los cuales son formulados en preguntas como las siguientes: ¿existe en criollo la palabra "kalkáar" (calcar)? o ¿qué significa en criollo la palabra "gáato"( gato $\left.^{3}\right)$ ?

Es importante señalar que algunos términos de origen español eran considerados por los informantes formas alternativas o sinónimas de otros vocablos de un origen etimológico diferente, como en el ejemplo (1) mencionado arriba o como en estos otros casos en los que se preguntaba, ¿cómo se dice en criollo ratonera? -Ratuoníera, rat trap, ous trap, o ¿cómo se dice portón?- Portóng, áyan duor.

En otras ocasiones, el vocablo de origen español era considerado por el informante la típica palabra criolla para designar el objeto o el evento en cuestión; e incluso, a veces, este afirmaba que era la única forma lingüística conocida por él: yúuka (yuca), dwéndi (duende), suorá $\left(\right.$ zorro $\left.^{4}\right)$. 


\subsection{Objetivo del trabajo}

Dada la gran cantidad de vocablos de origen español que han sido registrados, debido a la enorme influencia del español sobre el inglés criollo, a través de un extendido bilingüismo (cf. Herzfeld 1978, Winkler 1998), se plantea el problema de definir cuáles de estas palabras deben ser incluidas (como hispanismos) en el diccionario dialectal que se está elaborando.

Se ha considerado que la respuesta a este problema debe tomar al menos los siguientes aspectos téorico-metodológicos:

a. La distinción clara entre las nociones de préstamo léxico (loanword o lexical borrowing), mezcla de códigos (code mixing) y cambio de código (code switching).

b. La pertinencia de la función estandarizadora que atañería a este diccionario, según la definición tipológico-lexicográfica que le ha sido asignada.

c. La pertinencia funcional de los hispanismos para los destinatarios del diccionario.

\section{1. ¿Qué es un hispanismo en inglés criollo?}

Como ha sido mencionado anteriormente, la situación de un bilingüismo extendido en la gran mayoría de hablantes de inglés criollo y el prestigio preponderante del español, ha provocado una introducción más o menos masiva de vocabulario de origen español en el inglés criollo5. Sin embargo, hay que reconocer que no todo vocablo de "origen español" debe ser considerado un hispanismo propiamente dicho, si se distingue entre las situaciones lingüísticas de préstamo léxico, mezcla de códigos y cambio de códigos, todas las cuales son comunes precisamente en los casos de un contacto linguiístico un poco intenso (cf. la tipología de la intensidad del contacto lingüístico de Thomason \& Kaufman 1988, cap. 4).

El cambio de códigos consiste en intercambiar las lenguas coexistentes de manera que partes de una oración o proposición aparezcan en una lengua y otras en la otra lengua (Hock 1986: 479) 6 . El cambio de códigos es una estrategia lingüística de índole especialmente sintáctica. Algunos ejemplos de cambio de códigos recogidos en sesiones de trabajo con informante son los siguientes:

(3) Cuando estamos reunidos toda la familia, somos muchos, sólo falta una hermana, shi iz lívin in Stiets nou [ella está viviendo en Estados Unidos ahora], y nos reunimos...

(4) Di wie a spíikin [la forma de hablar] no me interesa.

(5) ¿Qué tal entonces dat ai av laik se, mm. [que yo tenga como digamos, este,] un pequeño bazar en mi casa, bot ai luk úova mai ous [pero yo me encargo de mi casa]?

(6) A óuswaif, pero a óuswaif, wat kain a óuswaif? [una ama de casa, pero una ama de casa, ¿qué clase de ama de casa?] Puede ser, digamos, que ella está en la casa, pero tiene una mied que le haga el oficio, y yo soy gerente de mi casa. 
(7) Eso de ir a medias en el márij, mi no plie wid dat [yo no juego con eso]. Eso sí que no, eso no es a medias.

En estos casos, obviamente, el intercambio de lenguas no significa presencia de hispanismos en inglés o de anglicismos en español.

Por otro lado, sin embargo, los vocablos que aparecen en cursiva en (6) y (7) representan ejemplos de mezcla de códigos. A diferencia del cambio de códigos que funciona en el nivel sintáctico, la mezcla de códigos opera un intercambio de índole léxica. La mezcla de códigos consiste, pues, en la inserción de vocablos de una lengua en la estructura gramatical de la otra (Hock 1986: 480).

En los ejemplos mencionados las palabras mied y márij no pueden ser consideradas anglicismos en español, sino palabras inglesas usadas en oraciones españolas. Si esto es así, debería considerarse lo mismo el caso de la palabra pero, la cual aparece en (6) en la oración de inglés criollo; es decir, que no es un hispanismo en criollo, sino una palabra española inserta en una proposición inglesa.

Sin embargo, la cosa no siempre es tan clara y distinta. Otros ejemplos de oraciones muestran que muchas veces es muy difícil distinguir entre mezcla de códigos y préstamo léxico.

Considérense los siguientes ejemplos:

(8) Go uol di bíebi, bikáa im gwain subíir di gráadas an drap.

ir sostener el bebé, porque él FUT subir la grada y caer

Sostenga al bebé porque va a subir las gradas y se va a caer.

(9) Wai yu arankáar di úoa dem out a di buk? por-qué usted arrancar la hoja PLU fuera de el libro ¿Por qué arrancó las hojas del libro?

(10) -Yu bring yu egzám?

- No, di tíicha no kalifikáar dem yet.

- usted traer su examen?

— no, la maestra no calificar ellos todavía

- ¿Trajo los exámenes?

- No, la maestra no los ha calificado todavía.

(11) Bring yu skuul buk fi du yu taréa.

Traer su escuela libro COM hacer su tarea

Traiga su cuaderno para que haga la tarea.

A primera vista pareciera que todas estas oraciones presentan también ejemplos de mezcla de códigos. En el ejemplo (8), tanto subíir como gráadas podrían fácilmente ser consideradas palabras españolas intrusas en el texto inglés, ya que el verbo infinitivo sería lo esperado después del auxiliar y el nombre aparece en plural también según lo esperado en español. Además, existen sinónimos de ambas palabras en inglés criollo, como go op o riez para subir y step, stier e incluso gried para grada. 
Por otro lado, las oraciones (9) y (10) presentarían, a primera vista, mejores candidatos de préstamos léxicos. Los verbos arankáar y kalifikáar aparecen sin conjugar y el nombre úoa se presenta en singular, pluralizado por medio del marcador morfológico correspondiente. Además, este último ha sufrido un obvio proceso de nativización fonológica.

La nativización tanto morfológica como fonológica ha sido aducida como una señal de que un vocablo ha sido incorporado como préstamo léxico en una lengua. A pesar de todo, habría que decir, por lo menos de úoa, que este no es de ningún modo el término más común de designar hoja en inglés criollo. Este es más bien liif.

La palabra taréa en la oración (11), de acuerdo con lo mencionado anteriormente parecería ser un caso de mezcla de códigos más que de préstamo léxico, ya que aparentemente tiene una forma muy semejante a la palabra española original tanto fonológica como morfológicamente. Sin embargo, las evidencias parecen señalar que este vocablo es un préstamo léxico de larga tradición. Al igual que otros vocablos del campo léxico escolar como kompanyéro y lapisíero, taréa es el término típico para designar el deber escolar. Es decir, que las palabras inglesas estándar homework, classmate y pen simplemente no se usarían en inglés criollo.

Según lo anterior, el criterio de frecuencia de uso y aceptabilidad del vocablo sería crucial para determinar que una palabra española sea un préstamo léxico. Sin embargo, aquí ya cabría preguntarse cómo podría el lexicógrafo determinar si estos criterios se aplican o no a un determinado caso.

Las siguientes oraciones muestran lo difícil y arbitrario que resulta a veces tomar una decisión.

(12) Di kampáana tokáar bai di skúul arédi.

la campana tocar por la escuela ya

Ya tocaron la campana de la escuela.

(13) Uuch líibro yu waan?

cuál libro usted quiere

¿Cuál libro quiere?

(14) Go botáar di basúura

ir botar la basura

Vaya bote la basura.

¿Por qué considerar que taréa y lapisiéro son préstamos, pero líibro y boradúor no? ¿Quién tiene el derecho de decidir y con base en qué criterios?

\section{Definición tipológica del diccionario}

Este ha sido concebido inicialmente como un diccionario trilingüe (de índole más pasiva que activa por estar dirigido sobre todo a hablantes nativos de inglés criollo, y a nativos y no nativos de inglés estándar), semasiológico (opuesto a onomasiológico), descriptivo (opuesto a prescriptivo), sincrónico (opuesto a diacrónico) pero que provee etimologías, para no especialistas (es 
decir, para un público sin preparación linguiística), de uso general (opuesto a técnico) y dialectológico (ya que es un diccionario especial restringido por criterios geográficos).

El diccionario ha sido pensado para describir el uso lingüístico real de los hablantes de la lengua, por ello es esencialmente descriptivo y no prescriptivo. Sin embargo, debido a que será el primer diccionario de esta variedad lingüística, no se puede soslayar el hecho de que este cumplirá una cierta función estadandarizadora y, por tanto, en cierta medida, también prescriptiva. Por esta razón, es importante determinar la naturaleza de los términos de origen español que se incluirían eventualmente en él.

Sobre este respecto, el investigador deberá tomar alguna de las siguientes decisiones, teóricamente posibles, que determinarían el ingreso de los vocablos de origen español como entradas del diccionario.

Por un lado, podría decidir, como opción extrema, no incluir ningún término de origen español en el diccionario. Este ha sido el caso, por ejemplo, del diccionario nahuatl - inglés de Karttunen (1983). Esta determinación, sin embargo, sería contradictoria con las caracterizaciones tipológicas atribuidas a este diccionario de ser de uso general, dialectológico y esencialmente descriptivo. En primer lugar, se estarían excluyendo arbitrariamente términos de uso claramente general, basado el investigador en un conocimiento etimológico del que carece por supuesto muchas veces el hablante ingenuo de la lengua. Así, al excluir todo hispanismo, habría que excluir palabras como dwéndi (duende), buóli (boli) y yúuka (yuca), a pesar de que no hay otros términos conocidos para designar los referentes mencionados.

Además, la exclusión de hispanismos también contradice el carácter dialectológico del diccionario, negando una característica claramente contrastiva del inglés de América Central respecto del llamado inglés estándar internacionalmente aceptado: la influencia del español.

Por último, un diccionario descriptivo no debería negar la inclusión de vocablos de uso normal por criterios de índole más bien prescriptiva, como la naturaleza etimológica de ellos.

Por otro lado, podría decidir, como otra opción extrema, incluir en el diccionario cualquier vocablo usado por los informantes, independientemente del origen etimológico de estos. Este parece que ha sido el caso de la mayoría de los diccionarios descriptivos dialectológicos (como en el caso del Dictionary of Caribbean English Usage de Allsopp 1996).

Esta opción, aunque aparentemente atractiva por su sencillez, acarrea muchos problemas al lexicógrafo, ya que, como se mencionó antes (cf. apartado 1), llevaría a incluir como hispanismos téminos que en realidad no lo son, pues simplemente representan casos de mezcla de códigos y no casos de adopción léxica. Desde una posición extrema, habría que reconocer que cualquier palabra española representa potencialmente un caso para ser incluido en el diccionario si algún informante decide usarla espontáneamente cuando hable en criollo.

Por último, una opción intermedia es decidir incluir en el diccionario solamente los vocablos que sean usados (y aceptados como palabras nativizadas) por un cierto grupo de informantes con ciertas características. Este ha sido el caso del diccionario de krio - inglés de Fyle \& Jones (1980), quienes incluyen como entrada todos los vocablos usados y sancionados por los informantes cuya edad oscilaba entre 35 y 60 años.

Nuestra práctica de trabajo con informantes ha mostrado, sin embargo, que los informantes no tienen criterios claros y uniformes para determinar la aceptación de un término de origen español como una palabra del inglés criollo. Además, todavía es menos probable lograr un consenso objetivo por parte de todos los informantes o jueces respecto de la nativización 
de ciertos téminos. En un caso, por ejemplo, respecto de las partes de la cara, un informante afirmaba que frénte sí es un préstamo, pero naríis no; búoka sí, pero láabyo no, según su opinión por supuesto, a pesar de que todos los términos pueden ser usados en una conversación normal en criollo.

\section{Los destinatarios}

Los destinatarios son hablantes nativos de inglés criollo, hablantes nativos de inglés estándar y hablantes nativos de español, especialmente costarricenses, que hablan inglés estándar.

Las necesidades por satisfacer en los usuarios son las siguientes:

- significado (provista en la equivalencia o la definición)

- pronunciación (provista en la entrada)

- ortografía inglesa estándar (provista en la etimología)

- sinónimos (provistos en la referencia cruzada)

- uso gramatical o uso en contexto (provisto en los ejemplos)

- estilo de la lengua

Dos ejemplos de entradas son los siguientes:

$\operatorname{bod}(<\mathrm{E} / \mathrm{I}$ bird [cal. S/E (C.R.)] ) n. (derog.) homosexual $\| n$. (desp.) homosexual.

búoli ( $<\mathrm{S} / \mathrm{E}$ boli ) $n$. frozen sweet made of water, sugar and food coloring, which is packed in cilinder-shaped plastic bags $\| n$. golosina congelada a base de agua, dulce y colorante que se empaca en bolsas plásticas de forma cilíndrica, boli (C.R.).

La cuestión de la pertinencia de la inclusión de hispanismos en el diccionario de acuerdo con los usuarios a los cuales va dirigido también es difícil de definir, debido a que se trata de tres grupos lingüísticos bastante disímiles.

Para el caso de los hablantes nativos de inglés criollo de Costa Rica y para los hablantes nativos de español, especialmente de Costa Rica, podría no ser necesaria la inclusión de hispanismos en el diccionario, puesto que su dominio del español les permitiría la comprensión de estos, incluso en casos extremos de mezcla de códigos como el siguiente:

$$
\begin{aligned}
& \text { A get a chichúota }{ }^{7} \text { pan mi frénte. } \\
& \text { yo recibir un chichón en mi frente } \\
& \text { Me hice un chichón en la frente. }
\end{aligned}
$$

Por otro lado, sin embargo, para hablantes nativos de inglés estándar (e incluso para hablantes nativos de español pero no costarricenses) parece imprescindible la inclusión de los hispanismos en el diccionario.

De cualquier forma, lo que está claro es que la inclusión o la exclusión de hispanismos en el diccionario definirá más bien el espectro de sus destinatarios. 


\section{Conclusiones}

Las reflexiones anteriores constituyen un preámbulo de discusión sobre el tema de la posible y adecuada inclusión de hispanismos en el diccionario de inglés criollo. Hasta este momento, no se ha decidido definitivamente cuál será la opción que se seguirá al respecto. Sin embargo, sí se puede reconocer cierta ventaja al excluir (casi) todo hispanismo del diccionario. En primer lugar, de esta forma se evitaría la estandarización de extranjerismos, al no ser reconocidos en el diccionario como verdaderos "criollismos". Además, por otro lado, se estaría fomentando tácitamente el uso de palabras castizas, provenientes del inglés.

Por otro lado, en el caso de que se decidiera incluir hispanismos en el diccionario quedan por definir las estrategias que se utilizarían para distinguir entre la mezcla de códigos y el préstamo léxico.

\section{Abreviaturas}

$\begin{array}{lll}\text { cal. } & = & \text { calco semántico } \\ \mathrm{COM} & = & \text { completizador } \\ \mathrm{C} . \mathrm{R} . & = & \text { costarriqueñismo } \\ \text { derog. } & = & \text { derogatory } \\ \text { desp. } & = & \text { despectivo } \\ \mathrm{E} / \mathrm{I} & = & \text { english/inglés } \\ \mathrm{FUT} & = & \text { futuro } \\ \mathrm{INT} & = & \text { intensificador } \\ \mathrm{n} . & = & \text { noun/nombre } \\ \mathrm{PLU} & = & \text { plural } \\ \mathrm{PRO} & = & \text { progresivo } \\ \mathrm{S} / \mathrm{E} & = & \text { spanish/español }\end{array}$

\section{Notas}

1. Esta palabra es un costarriqueñismo que significa melancolía, tenue tristeza, añoranza, nostalgia (Quesada Pacheco 1991: 52).

2. Costarriqueñismo: Nombre de pájaro de grito estridente (Philorhinus morio) (Quesada Pacheco 1991: 52).

3. En español, esta palabra, además de designar al animal felino, se usa para referirse a un tipo de repostería comercial (este término probablemente sea un préstamo del francés 'gâteau' pastel).

4. Costarriqueñismo: Nombre de cuadrúpedo nocturno comestible, de poco pelaje y dientes fuertes (Didelphys marsupialis) (Quesada Pacheco 1991: 52).

5. Además de vocabulario, el inglés criollo ha adoptado algunos rasgos estructurales del español, como, por ejemplo, el dativo de interés:

Mi graní ded pan mi laas wiik.

mi abuela morir sobre mí última semana

Se me murió mi abuela la semana pasada. 
6. La traducción es mía.

7. Esta palabra es un costarriqueñismo.

\section{Bibliografía}

Allsopp, Richard. 1996. Dictionary of Caribbean English Usage. Oxford: Oxford University Press.

Fyle, Clifford \& Eldred Jones. 1980. A Krio-English dictionary. Oxford: Oxford University Press.

Herzfeld, Anita. 1978. "Vida o muerte en el criollo limonense". Revista de Filología y Lingüística. 4(2): 17-24.

Hock, Hans. 1986. Principles of Historical Linguistics. Berlin: Mouton de Gruyar.

Holm, John. 1978. The creole english of Nicaragua's miskito coast. London: University of London.

Karttunen, Frances. 1983. An analytical dictionary of Nahuatl. Austin.

Quesada Pacheco, Miguel Ángel. 1991. Nuevo diccionario de costarriqueñismos. Cartago: Editorial Tecnológica de Costa Rica.

Winkler, Elizabeth. 1998. Limonese Creole: A case of contact-induced language change. Bloomington: Indiana University.

Thomason, Sarah \& Terrence Kaufman. 1988. Language contact, creolization, and genetic linguistics. Berkeley: University of California Press. 